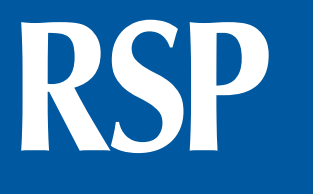

http://www.rsp.fsp.usp.br/
Revista de Saúde Pública

\section{Fatores associados à diarreia em menores de cinco anos, no estado de Pernambuco, segundo inquéritos realizados em 1997 e 2006}

\author{
Maria Josemere de Oliveira Borba Vasconcelos', Anete Rissin', José Natal Figueiroa', Pedro Israel \\ Cabral de Lira", Malaquias Batista Filho' \\ I Instituto de Medicina Integral Prof. Fernando Figueira. Diretoria de Pesquisas. Recife, PE, Brasil \\ " Universidade Federal de Pernambuco. Departamento de Nutrição. Recife, PE, Brasil
}

\section{RESUMO}

OBJETIVO: Descrever e comparar variações dos fatores associados à prevalência de diarreia em menores de cinco anos no estado de Pernambuco.

MÉTODOS: Foram utilizados os bancos de dados de dois inquéritos de base populacional nos anos de 1997 e 2006, com 2.078 e 1.650 crianças, respectivamente, avaliadas em 18 municípios de Pernambuco (Região Metropolitana do Recife, interior urbano e rural). As variáveis, alocadas em níveis hierárquicos, foram analisadas por meio de razões de prevalência e regressão de Poisson.

RESULTADOS: Apenas quatro variáveis se mostraram independentemente associadas e constaram no modelo final hierarquizado: área geográfica, número de pessoas por cômodo, idade materna e idade da criança. Em 1997: interior urbano = 1,33 (IC95\% 1,06-1,66), interior rural = 1,22 (IC95\% 0,97-1,53) e em 2006: interior urbano $=1,87($ IC95\% 1,31-2,66), interior rural $=2.07$ (IC95\% 1.50-2.85); número de pessoas por cômodo (1997): 1 a menos de 2 = 1,29 (IC95\% 0,98-1,68), dois ou mais = 1,47 (IC95\% 1,11-1,95) e em 2006: 1 a menos de $2=0.86$ (IC95\% 0,68-1,09), dois ou mais = 1,29 (IC95\% 0,94-1,75); idade materna (1997): 10 a 19 anos = 1,48 (IC95\% 1,05-2,08), 20 a 24 anos = 1,23 (IC95\% 0,94-1,60), 25 a 34 anos = 1,01 (IC95\% 0,78-1,30) e em 2006: 10 a 19 anos = 1,70 (IC95\% 1,08-2,66), 20 a 24 anos = 1,64 (IC95\% 1,16-2,32), 25 a 34 anos = 1,20 (IC95\% 0,89-1,62); e idade da criança (1997): $0-11$ meses $=1,57$ (IC95\% 1,27-1,94), 12-23 meses $=1,73$ $($ IC95\% 1,41-2,12) e em 2006: $0-11$ meses $=1,04$ (IC95\% 0,76-1,41), 12-23 meses $=1,77$ (IC95\% $1,41-2,23)$.

CONCLUSÕES: Houve uma grande variabilidade dos condicionantes das diarreias em crianças entre os dois períodos analisados. Em nível de políticas públicas, apesar de mudanças em termos de pessoas, sequências temporais e espaços geográficos, as diarreias continuam em uma escala importante no elenco de prioridades do poder governamental.

DESCRITORES: Pré-Escolar. Diarreia Infantil, epidemiologia. Diarreia, epidemiologia. Prevalência. Fatores de Risco. Fatores Socioeconômicos. Estudos de Séries Temporais.

Copyright: Este é um artigo de acesso aberto distribuído sob os termos da Licença de Atribuição Creative Commons, que permite uso irrestrito, distribuição e reprodução em qualquer meio desde que o autor e a fonte originais sejam creditados.

Correspondência: Vasconcelos

Rua dos Coelhos, 300 Boa vista 50070-550 Recife, PE, Brasil

Recebido: 29 jun 2016

Como citar: Vasconcelos MJOB Rissin A, Figueiroa JN, Lira PIC, Batista Filho M. Fatores associados à diarreia em menores de cinco segundo inquéritos realizados em 1997 e 2006. Rev Saude Publica. 2018;52:48. 


\section{INTRODUÇÃO}

A doença diarreica continua sendo um dos principais problemas que acometem a população infantil nos primeiros anos de vida, notadamente nas regiões menos desenvolvidas. Por sua elevada morbimortalidade, caracteriza-se como uma questão prioritária de saúde pública ${ }^{1,2}$ e representa importante demanda junto à rede de serviços de saúde em escala mundial, regional e local ${ }^{3,4}$. Em vários países, a diarreia ainda aparece como a principal causa do óbito infantil, consistindo em um indicador discriminatório de espaços geográficos caracterizados por condições precárias de vida coletiva, tipificando os chamados ecossistemas de pobreza ${ }^{1,5}$.

O problema está relacionado com fatores de ordem ambiental, socioeconômica, cultural, baixa cobertura e efetividade dos serviços de saúde. Esses fatores podem estabelecer diferenças marcantes na sua evolução em função das desigualdades que comprometem o perfil de produção e distribuição de bens e serviços no contexto de diferentes estratos da população ${ }^{6,7}$.

Em função de suas características clínicas e epidemiológicas, as diarreias podem figurar como "condições traçadoras" ${ }^{\circ}$, uma vez que o acompanhamento descritivo e analítico de sua evolução temporal e espacial, mais do que a demarcação de uma nosologia específica, pode representar a cartografia do subdesenvolvimento e da pobreza, caracterizado fundamentalmente pela ocorrência de doenças evitáveis e curáveis.

Esses aspectos conceituais e empíricos justificam o interesse de avaliações contínuas ou periódicas sobre as tendências históricas, geográficas e seus fatores de risco, de modo a representar o grau de desenvolvimento humano da população e dos serviços e ações de saúde sob uma perspectiva territorial e temporal. Sob o ponto de vista epidemiológico, a configuração do complexo de fatores significativamente correlacionados com sua ocorrência - incluindo eventos intermediários, como a hospitalização, ou finais, como a mortalidade específica - oferece um quadro de referências importantes para os desafios políticos e programáticos do setor saúde.

A realização de estudos periódicos de base populacional no estado de Pernambuco sobre problemas de saúde materno-infantil proporciona em particular a avaliação das grandes mudanças nos perfis de morbidade a partir de 1990, quando se configura o processo mais dinâmico da chamada transição epidemiológica ${ }^{9,10}$. Recorrendo ao estudo inicial (1991) sobre saúde materno-infantil, a prevalência de diarreia (22,5\%) em crianças em Pernambuco já era bem superior aos valores encontrados no conjunto dos estados do Nordeste $(14,4 \%)$ e da região Sudeste $(8 \%)^{11}$. Esta abordagem se destaca quando se incorpora uma visão multifatorial do contexto, ou seja, uma perspectiva holística de saúde ${ }^{12}$. É pertinente considerar que em breve deverá ser realizada outra avaliação de base populacional sobre os problemas e demandas de serviços de saúde no estado de Pernambuco, a IV PESN, de modo a atualizar as informações sobre a evolução de vários problemas de saúde materno-infantil, inclusive sobre o comportamento das diarreias e sua participação na composição das demandas ambulatoriais, hospitalizações e causas de morte mais importantes.

Objetiva-se, assim, descrever e comparar variações dos fatores associados à diarreia em menores de cinco anos no estado de Pernambuco. Para tanto, foi realizado um inventário descritivo e analítico das doenças diarreicas em crianças desse estado, com base em dois inquéritos de campo realizados nos anos de 1997 e 2006, como partes do projeto institucional mais abrangente que deve ser apreciado nos próximos anos a fim de focalizar tendências espaciais e temporais de ocorrência do problema, bem como a determinação de seus possíveis fatores de risco.

\section{MÉTODOS}

Este estudo transversal de base populacional utilizou dados secundários das II e III Pesquisas Estaduais de Saúde e Nutrição (II e III PESN), realizadas em 1997 e 2006, representando os 
estratos urbano (Região Metropolitana do Recife e interior urbano [IU]) e rural (interior rural [IR]) da população, tendo como objetivo a atualização e ampliação do diagnóstico de saúde, nutrição, alimentação, demandas de serviços e condições socioeconômicas da população do estado de Pernambuco ${ }^{10,13}$ população de estudo foi constituída por menores de cinco anos, incluídas nos dois inquéritos supracitados, com 2.078 e 1.650 crianças, avaliadas nos anos de 1997 e 2006, respectivamente.

O processo de amostragem desses inquéritos foi do tipo probabilístico e estratificado em três estágios. Inicialmente, constou de um sorteio dos municípios, depois dos setores censitários e por fim dos domicílios. Os 18 municípios incluídos nas II e III PESN foram: Recife, Olinda, Paulista, Jaboatão, Cabo, São Bento do Una, Goiana, Itaíba, Belém do São Francisco, Orobó, Caruaru, Camocim de São Félix, Triunfo, Bodocó, Palmares, Ribeirão, Panelas e Itaquitinga.

As entrevistas foram realizadas com a mãe ou o responsável pela criança, aplicando-se formulários e questionários compostos por perguntas pré-codificadas contendo informações socioeconômicas, ambientais, demográficas, biológicas, características maternas e acesso aos serviços de saúde. A variável dependente foi representada pela morbidade por diarreia, referenciada nas duas últimas semanas que antecederam a pesquisa. Definiu-se como caso, a ocorrência de três ou mais evacuações diárias, de consistência líquida ou semilíquida, acompanhadas ou não de muco ou sangue ${ }^{14}$.

As variáveis independentes foram agrupadas em: a) fator geográfico (urbano e rural); b) fatores ambientais (abastecimento de água, esgotamento sanitário, tratamento da água de beber, destino do lixo, tipo de piso, tipo de parede, número de pessoas por cômodo e número de pessoas por dormitório); c) fatores socioeconômicos (posse de geladeira, visita do agente comunitário de saúde e renda familiar per capita); d) fatores maternos (idade, condição de trabalho e escolaridade) e; e) fatores biológicos (peso ao nascer, estado nutricional e amamentação).

O estado nutricional das crianças foi analisado por meio de antropometria, utilizando-se os indicadores peso para a idade, estatura para a idade e peso para a estatura, segundo a distribuição em escores Z. Para a classificação dos dados antropométricos, foi utilizado o padrão de referência adotado pelo Ministério da Saúde ${ }^{15}$, sendo empregado o software Anthro, versão 3.2.2 $2^{16}$.

Para identificar fatores associados à ocorrência de diarreia, tanto em 1997 quanto em 2006, as variáveis preditoras foram hierarquizadas em três níveis por critérios de lógica conceitual (Figura). A construção do modelo hierarquizado usado neste estudo teve como referência a modelagem aplicada por Fuchs et al. para a investigação de fatores de risco de diarreia grave ${ }^{17}$. Como variáveis de controle, foram selecionadas as variáveis sexo e idade, que, pelo seu significado biológico e sem receber influência de outras variáveis, poderiam interferir diretamente na determinação do problema investigado. A partir dessa disposição por níveis, utilizamos um processo de análise univariada reunindo as categorias de variáveis em cada grupo mediante cálculo de razões de prevalência (RP) e respectivos intervalos de confiança (IC95\%) e adotando valores de p abaixo de 0,25 como de significância estatística. As variáveis com esse valor foram admitidas para integrar a sequência de análises multivariadas (regressão de Poisson), partindo do nível mais distal (categorias geográficas) para as mais proximais (variáveis referentes à criança). Em cada um dos níveis seguintes, foi ajustado o modelo de regressão múltipla de Poisson com as variáveis do nível correspondente e variáveis estatisticamente significativas dos grupos anteriores. O modelo final foi composto pelas variáveis próprias do nível que apresentaram valor $\mathrm{p}<0,05$, bem como pelas demais variáveis dos níveis anteriores na mesma condição. Os ajustes dos modelos levaram em consideração o efeito de clusters presente nos dados. Foi empregada a estratégia purposeful selection para a escolha das variáveis explanatórias dos modelos de cada nível. A análise de regressão de Poisson foi feita no software Stata 12.1SE. 
Nível distal

Grupo A

Fator geográfico

Área geográfica

Nível intermediário

Grupo B

Fatores ambientais

\begin{tabular}{|c|}
\hline Abastecimento de água \\
Esgotamento sanitário \\
Tratamento da água de beber \\
Destino do lixo \\
Tipo de piso \\
Tipo de parede \\
Número de pessoas por cômodo \\
Número de pessoas por dormitório \\
\hline
\end{tabular}

Grupo C

Fatores socioeconômicos

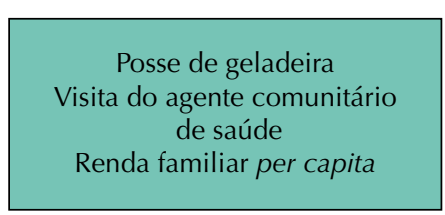

Nível proximal

Grupo E

Fatores biológicos

Peso ao nascer

Estado nutricional (antropometria)

Amamentação

Diarreia em menores

de cinco anos

Figura. Modelo causal hierarquizado dos possíveis fatores associados à diarreia em menores de cinco anos no estado de Pernambuco nos anos 1997 e 2006.

Cabe ressaltar que a vacina rotavírus começou a ser aplicada (crianças) no início do último inquérito de campo (março a maio), de modo que seu impacto ainda não seria detectável em nível populacional.

Os inquéritos (II e III PESN) foram aprovados pelo Comitê de Ética em Pesquisa em Seres Humanos do Centro de Ciências da Saúde da Universidade Federal de Pernambuco (II PESN 27/2/1997) e do Instituto de Medicina Integral Professor Fernando Figueira (III PESN - 9/11/2005).

\section{RESULTADOS}

A prevalência das diarreias baixou de 19,8\% (1997) para 18,1\% (2006). Em relação ao Grupo A ( fatores geográficos), ressalta-se a notável redução que houve na Região Metropolitana do Recife, passando de 16,9\% para 10,5\%. Como fontes de associação estatisticamente significantes com as diarreias, verifica-se que os estratos geográficos se apresentaram nos dois inquéritos, com os menores riscos na Região Metropolitana do Recife (Tabela 1). No Grupo B (fatores ambientais), composto por oito variáveis, o esgotamento sanitário, o destino do lixo, o número de pessoas por cômodo da moradia e o tratamento da água de beber foram fatores comuns de risco nos anos analisados. Já o abastecimento de água da rede pública, bem como o tratamento da água de beber foram estatisticamente significativos apenas nas análises univariadas referentes ao ano de 2006. No grupo C ( fatores socioeconômicos), a posse de geladeira apresenta-se como um fator de proteção da ocorrência de diarreia em 1997 e 2006, do mesmo modo que a renda familiar per capita. Das variáveis incluídas no grupo D (fatores maternos), baixas idade e escolaridade maternas comportaram-se como fatores de risco de diarreia em seus filhos, nos dois anos avaliados. 
Tabela 1. Análises univariadas dos fatores associados à ocorrência de diarreia em menores de cinco anos. Pernambuco, 1997 e 2006.

\begin{tabular}{|c|c|c|c|c|c|c|c|c|}
\hline \multirow{3}{*}{ Variável } & \multicolumn{4}{|c|}{1997} & \multicolumn{4}{|c|}{2006} \\
\hline & Amostra & Diarreia & \multirow{2}{*}{ RP (IC95\%) } & \multirow{2}{*}{$\mathbf{p}$} & \multirow{2}{*}{$\frac{\text { Amostra }}{N}$} & \multirow{2}{*}{$\begin{array}{c}\text { Diarreia } \\
\mathbf{n}(\%)\end{array}$} & \multirow{2}{*}{ RP (IC95\%) } & \multirow{2}{*}{$\mathbf{p}$} \\
\hline & $\mathbf{N}$ & n (\%) & & & & & & \\
\hline \multicolumn{9}{|c|}{ Nível distal } \\
\hline \multicolumn{9}{|l|}{ Grupo A } \\
\hline \multicolumn{9}{|l|}{ Área geográfica } \\
\hline Região metropolitana & 734 & $124(16,9)$ & 1,0 & 0,043 & 427 & $45(10,5)$ & 1,0 & $<0,001$ \\
\hline Interior urbano & 685 & $154(22,5)$ & $1,33(1,06-1,66)$ & & 416 & $81(19,5)$ & $1,87(1,31-2,66)$ & \\
\hline Interior rural & 651 & $131(20,1)$ & $1,22(0,97-1,53)$ & & 789 & $169(21,4)$ & $2,07(1,50-2,85)$ & \\
\hline \multicolumn{9}{|c|}{ Nível intermediário } \\
\hline \multicolumn{9}{|l|}{ Grupo B } \\
\hline \multicolumn{9}{|l|}{ Abastecimento de água } \\
\hline Rede geral & 1.359 & $260(19,1)$ & 1,0 & 0,243 & 918 & $148(16,1)$ & 1,0 & 0,018 \\
\hline Outro & 711 & $149(21,0)$ & $1,12(0,93-1,35)$ & & 714 & $147(20,6)$ & $1,29(1,04-1,60)$ & \\
\hline \multicolumn{9}{|l|}{ Esgotamento sanitário } \\
\hline Rede pública & 554 & $95(17,1)$ & 1,0 & 0,004 & 547 & $83(15,2)$ & 1,0 & 0,045 \\
\hline Fossa com tampa & 724 & $130(18,0)$ & $1,05(0,82-1,35)$ & & 519 & $93(17,9)$ & $1,18(0,90-1,56)$ & \\
\hline Outro & 792 & $184(23,2)$ & $1,40(1,11-1,76)$ & & 563 & $119(21,1)$ & $1,39(1,07-1,81)$ & \\
\hline \multicolumn{9}{|c|}{ Tratamento da água de beber } \\
\hline Rede geral & 1.207 & $209(17,3)$ & 1,0 & $<0,001$ & 823 & $104(12,6)$ & 1,0 & $<0,001$ \\
\hline Outro & 863 & $200(23,2)$ & $1,34(1,12-1,61)$ & & 809 & $191(23,6)$ & $1,78(1,35-2,35)$ & \\
\hline \multicolumn{9}{|l|}{ Destino do lixo } \\
\hline Coleta pública & 1.046 & $177(16,9)$ & 1,0 & $<0,001$ & 945 & $151(16,0)$ & 1,0 & 0,008 \\
\hline Outra forma & 1.024 & $232(22,7)$ & $1,37(1,14-1,65)$ & & 687 & $144(21,0)$ & $1,33(1,08-1,65)$ & \\
\hline Tipo de piso & & & & & & & & \\
\hline Cerâmica/Cimento & 1.780 & $343(19,3)$ & 1,0 & 0,183 & 1.544 & $277(17,9)$ & 1,0 & 0,537 \\
\hline Outros & 290 & $66(22,8)$ & $1,18(0,92-1,51)$ & & 88 & $18(20,4)$ & $1,14(0,75-1,74)$ & \\
\hline Tipo de parede & & & & & & & & \\
\hline Alvenaria/Tijolo & 1.771 & $347(19,6)$ & 1,0 & 0,544 & 1.525 & $273(17,9)$ & 1,0 & 0,550 \\
\hline Outro & 299 & $62(20,7)$ & $1,08(0,84-1,39)$ & & 107 & $22(20,6)$ & $1,14(0,75-1,72)$ & \\
\hline $\mathrm{N}^{\circ}$ de pessoas por cômoc & & & & & & & & \\
\hline$<1$ & 429 & $63(14,7)$ & 1,0 & 0,004 & 653 & $113(17,3)$ & 1,0 & 0,015 \\
\hline $1-2$ & 1.014 & $200(19,7)$ & $1,34(1,03-1,75)$ & & 780 & $132(16,9)$ & $0,96(0,76-1,21)$ & \\
\hline$\geq 2$ & 627 & $146(23,3)$ & $1,59(1,21-2,10)$ & & 199 & $50(25,1)$ & $1,45(1,08-1,95)$ & \\
\hline $\mathrm{N}^{\circ}$ de pessoas por dormit & & & & & & & & \\
\hline$<2$ & 364 & $54(14,8)$ & 1,0 & 0,022 & 334 & $52(15,6)$ & 1,0 & 0,405 \\
\hline $2-3$ & 675 & $131(19,4)$ & $1,31(0,97-1,75)$ & & 599 & $108(18,0)$ & $1,15(0,85-1,57)$ & \\
\hline$\geq 3$ & 1.031 & $224(21,7)$ & $1,46(1,11-1,92)$ & & 699 & $135(19,3)$ & $1,23(0,91-1,65)$ & \\
\hline Grupo C & & & & & & & & \\
\hline Posse de geladeira & & & & & & & & \\
\hline $\operatorname{Sim}$ & 1.138 & $178(15,6)$ & 1,0 & $<0,001$ & 1.105 & $179(16,2)$ & 1,0 & 0,007 \\
\hline Não & 932 & $231(24,8)$ & $1,58(1,32-1,90)$ & & 527 & $116(22,0)$ & $1,35(1,09-1,68)$ & \\
\hline Visita do agente de saúde & & & & & & & & \\
\hline Sim & 680 & $151(22,2)$ & 1,0 & 0,081 & 1.353 & $248(18,3)$ & 1,0 & 0,672 \\
\hline Não & 1.377 & $256(18,6)$ & $0,85(0,70-1,02)$ & & 276 & $47(17,0)$ & $0,94(0,71-1,24)$ & \\
\hline Renda familiar per capita & & & & & & & & \\
\hline$<0,50$ & 1.253 & $285(22,7)$ & $1,50(1,22-1,83)$ & $<0,001$ & 1.366 & $267(19,5)$ & $2,18(1,44-3,32)$ & $<0,001$ \\
\hline$\geq 0,50$ & 798 & $122(15,3)$ & 1,0 & & 233 & $21(9,0)$ & 1,0 & \\
\hline Grupo D & & & & & & & & \\
\hline Idade materna (anos) & & & & & & & & \\
\hline $10-19$ & 170 & $47(27,6)$ & $1,58(1,12-2,23)$ & 0,002 & 100 & $24(24,0)$ & $1,64(1,06-2,52)$ & 0,009 \\
\hline $20-24$ & 568 & $131(23,1)$ & $1,31(1,00-1,72)$ & & 363 & $83(22,9)$ & $1,57(1,15-2,14)$ & \\
\hline $25-34$ & 922 & $159(17,2)$ & $0,97(0,75-1,26)$ & & 795 & $134(16,9)$ & $1,15(0,86-1,55)$ & \\
\hline$\geq 35$ & 408 & $72(17,6)$ & 1,0 & & 371 & $54(14,6)$ & 1,0 & \\
\hline
\end{tabular}

Continua 
Tabela 1. Análises univariadas dos fatores associados à ocorrência de diarreia em menores de cinco anos. Pernambuco, 1997 e 2006. Continuação

\begin{tabular}{|c|c|c|c|c|c|c|c|c|}
\hline \multicolumn{9}{|l|}{ Condição de trabalho da mãe } \\
\hline Trabalha & 610 & $98(16,1)$ & 1,0 & 0,010 & 132 & $24(18,2)$ & 1,0 & 0,923 \\
\hline Não trabalha & 1.456 & $310(21,3)$ & $1,32(1,07-1,64)$ & & 1.496 & $270(18,0)$ & $0,98(0,67-1,43)$ & \\
\hline \multicolumn{9}{|l|}{ Escolaridade materna (anos) } \\
\hline$<4$ & 863 & $205(23,8)$ & $2,05(1,57-2,67)$ & $<0,001$ & 524 & $110(21,0)$ & $1,47(1,11-1,94)$ & 0,028 \\
\hline $4-7$ & 644 & $138(2,14)$ & $1,85(1,40-2,44)$ & & 623 & $117(18,8)$ & $1,31(0,99-1,74)$ & \\
\hline$\geq 8$ & 547 & $63(11,5)$ & 1,0 & & 474 & $67(14,1)$ & 1,0 & \\
\hline \multicolumn{9}{|c|}{ Nível proximal } \\
\hline \multicolumn{9}{|l|}{ Grupo E } \\
\hline \multicolumn{9}{|l|}{ Idade da criança(meses) } \\
\hline $0-11$ & 458 & $114(24,9)$ & $1,63(1,32-2,01)$ & $<0,001$ & 305 & $52(17,0)$ & $1,14(0,85-1,53)$ & $<0,001$ \\
\hline $12-23$ & 413 & $112(27,1)$ & $1,77(1,45-2,17)$ & & 356 & $100(28,1)$ & $1,92(1,54-2,41)$ & \\
\hline $24-59$ & 1.199 & $183(1,53)$ & 1,0 & & 971 & $143(14,7)$ & 1,0 & \\
\hline \multicolumn{9}{|l|}{ Sexo } \\
\hline Masculino & 1.028 & $206(20,0)$ & $1,03(0,87-1,23)$ & 0,725 & 841 & $161(19,1)$ & $1,13(0,92-1,40)$ & 0,251 \\
\hline Feminino & 1.042 & $203(19,5)$ & 1,0 & & 791 & $134(16,9)$ & 1,0 & \\
\hline \multicolumn{9}{|l|}{ Peso ao nascer (grama) } \\
\hline$<2.500$ & 150 & $36(24,0)$ & $1,14(0,83-1,56)$ & 0,682 & 138 & $20(14,5)$ & $0,80(0,52-1,22)$ & 0,503 \\
\hline $2.500-2.999$ & 373 & $74(19,8)$ & $0,98(0,77-1,23)$ & & 294 & $57(19,4)$ & $1,06(0,81-1,39)$ & \\
\hline$\geq 3.000$ & 1.377 & $282(20,5)$ & 1,0 & & 1.148 & $207(18,0)$ & 1,0 & \\
\hline \multicolumn{9}{|l|}{ Estatura para idade } \\
\hline Muito baixa/Baixa & 238 & $64(26,9)$ & $1,38(1,09-1,75)$ & 0,007 & 138 & $31(22,5)$ & $1,24(0,89-1,73)$ & 0,195 \\
\hline Adequada & 1.757 & $335(19,1)$ & 1,0 & & 1.445 & $257(17,8)$ & 1,0 & \\
\hline \multicolumn{9}{|l|}{ Peso para estatura } \\
\hline Magreza acentuada/Magreza & 36 & $6(16,7)$ & $0,92(0,47-1,81)$ & 0,007 & 25 & $4(16,0)$ & $0,84(0,36-2,00)$ & 0,303 \\
\hline Eutrofia & 1.473 & $268(18,2)$ & 1,0 & & 1.092 & $207(19,0)$ & 1,0 & \\
\hline $\begin{array}{l}\text { Risco de sobrepeso/Sobrepeso/ } \\
\text { Obesidade }\end{array}$ & 512 & $127(24,8)$ & $1,35(1,12-1,62)$ & & 467 & $75(16,1)$ & $0,82(0,64-1,06)$ & \\
\hline \multicolumn{9}{|l|}{ Peso para idade } \\
\hline Muito baixo/Baixo & 100 & $23(23,0)$ & $1,19(0,81-1,74)$ & 0,380 & 51 & $14(27,5)$ & $1,48(0,92-2,38)$ & 0,103 \\
\hline Adequado/Elevado & 1.933 & $381(19,7)$ & 1,0 & & 1.540 & $275(17,9)$ & 1,0 & \\
\hline \multicolumn{9}{|l|}{ Amamentação } \\
\hline Mama/Mamou & 1.840 & $363(19,7)$ & 1,0 & 0,886 & 1.542 & $279(18,1)$ & 1,0 & 0,903 \\
\hline Nunca mamou & 215 & $42(19,5)$ & $0,98(0,73-1,32)$ & & 81 & $15(18,5)$ & $1,03(0,64-1,65)$ & \\
\hline
\end{tabular}

SM: salário mínimo

Os resultados do modelo final hierarquizado para os fatores associados à ocorrência de diarreia, encontram-se na Tabela 2. De um total de 10 variáveis, apenas quatro se mostraram independentemente associadas: área geográfica, número de pessoas por cômodo, idade materna e idade da criança. Os resultados são apresentados a seguir, com as respectivas razões de prevalência e intervalos de confiança. Em 1997: IU = 1,33 (IC95\% 1,06-1,66), IR = 1,22 (IC95\% 0,97-1,53) e ocorrência de diarreia (2006): IU = 1,87 (IC95\% 1,31-2,66), IR(2006) = 2,07 (IC95\% 1,50-2,85); número de pessoas por cômodo (1997): 1 a menos de $2=1.29$ (IC95\% 0,98-1,68), dois ou mais = 1,47 (IC95\% 1,11-1,95) e número de pessoas por cômodo (2006): 1 a menos de 2 = 0,86 (IC95\% 0,68-1,09), dois ou mais = 1,29 (IC95\% 0,94-1,75); idade materna (1997): 10 a 19 anos $=1,48$ (IC95\% 1,05-2,08), 20 a 24 anos = 1,23 (IC95\% 0,94-1,60), 25 a 34 anos = 1,01 (IC95\% 0,78-1,30) e idade materna (2006): 10 a 19 anos $=1,70$ (IC95\% 1,08-2,66), 20 a 24 anos = 1,64 (IC95\% 1,162,32), 25 a 34 anos = 1,20 (IC95\% 0,89-1,62); e idade da criança (1997): $0-11$ meses = 1,57 (IC95\% 1,27-1,94), 12-23 meses = 1,73 (IC95\% 1,41-2,12) e idade da criança (2006): $0-11$ meses $=1,04$ (IC95\% 0,76-1,41), 12-23 meses = 1,77 (IC95\% 1,41-2,23). 
Tabela 2. Modelos finais hierarquizados da diarreia em menores de cinco anos. Pernambuco, 1997 e 2006.

\begin{tabular}{|c|c|c|c|c|c|c|c|c|}
\hline \multirow{3}{*}{ Variável } & \multicolumn{4}{|c|}{1997} & \multicolumn{4}{|c|}{2006} \\
\hline & Amostra & Diarreia & \multirow{2}{*}{ RP $(\text { IC95\% } \%)^{a}$} & \multirow{2}{*}{$\mathbf{p}$} & Amostra & Diarreia & \multirow{2}{*}{$\operatorname{RP}(\mathrm{IC95} \%)^{\mathrm{a}}$} & \multirow{2}{*}{ p } \\
\hline & $\mathbf{N}$ & n (\%) & & & $\mathbf{N}$ & n (\%) & & \\
\hline \multicolumn{9}{|c|}{ Nível distal } \\
\hline \multicolumn{9}{|l|}{ Grupo A } \\
\hline \multicolumn{9}{|l|}{ Área geográfica } \\
\hline Região metropolitana & 734 & $124(16,9)$ & 1,0 & 0,043 & 427 & $45(10,5)$ & 1,0 & $<0,001$ \\
\hline Interior urbano & 685 & $154(22,5)$ & $1,33(1,06-1,66)$ & & 416 & $81(19,5)$ & $1,87(1,31-2,66)$ & \\
\hline Interior rural & 651 & $131(20,1)$ & $1,22(0,97-1,53)$ & & 789 & $169(21,4)$ & $2,07(1,50-2,85)$ & \\
\hline \multicolumn{9}{|c|}{ Nível intermediário } \\
\hline \multicolumn{9}{|l|}{ Grupo B } \\
\hline \multicolumn{9}{|l|}{ Destino do lixo } \\
\hline Coleta pública & 1.046 & $177(16,9)$ & 1,0 & $<0,001^{\mathrm{b}}$ & - & - & - & - \\
\hline Outra forma & 1.024 & $232(22,7)$ & $1,50(1,19-1,88)$ & & - & - & - & - \\
\hline \multicolumn{9}{|l|}{$N^{o}$ de pessoas por cômodo } \\
\hline$<1$ & 429 & $63(14,7)$ & 1,0 & $0,026^{b}$ & 653 & $113(17,3)$ & 1,0 & 0,028 \\
\hline 12 & 1.014 & $200(19,7)$ & $1,29(0,98-1,68)$ & & 780 & $132(16,9)$ & $0,86(0,68-1,09)$ & \\
\hline$\geq 2$ & 627 & $146(23,3)$ & $1,47(1,11-1,95)$ & & 199 & $50(25,1)$ & $1,29(0,94-1,75)$ & \\
\hline \multicolumn{9}{|l|}{ Abastecimento de água } \\
\hline Rede geral & - & - & - & - & 918 & $148(16,1)$ & 1,0 & 0,028 \\
\hline Outros & - & - & - & - & 714 & $147(20,6)$ & $0,67(0,47-0,96)$ & \\
\hline \multicolumn{9}{|l|}{ Tratamento da água de beber } \\
\hline Fervida/Filtrada/Clorada/Mineral & - & - & - & - & 823 & $104(12,6)$ & 1,0 & $<0,001$ \\
\hline Coada/Sem Tratamento/Outros & - & - & - & - & 809 & $191(23,6)$ & $1,77(1,35-2,32)$ & \\
\hline \multicolumn{9}{|c|}{ Nível distal } \\
\hline \multicolumn{9}{|l|}{ Grupo C } \\
\hline \multicolumn{9}{|l|}{ Posse de geladeira } \\
\hline $\operatorname{Sim}$ & 1.138 & $178(15,6)$ & 1,0 & $0,001^{c}$ & - & - & & - \\
\hline Não & 932 & $231(24,8)$ & $1,45(1,16-1,80)$ & & - & - & & - \\
\hline \multicolumn{9}{|l|}{ Renda per capita } \\
\hline$<0,50$ & - & - & - & - & 1.366 & $267(19,5)$ & & 0,026 \\
\hline$\geq 0,50$ & - & - & - & - & 233 & $21(9,0)$ & & \\
\hline \multicolumn{9}{|l|}{ Grupo D } \\
\hline Idade materna (anos) & & & & & & & & \\
\hline $10-19$ & 169 & $47(27,8)$ & $1,48(1,05-2,08)$ & $0,037^{\mathrm{d}}$ & 98 & $24(24,5)$ & & $0,014^{\mathrm{d}}$ \\
\hline $20-24$ & 561 & $129(23,0)$ & $1,23(0,94-1,60)$ & & 344 & $78(22,7)$ & & \\
\hline $25-34$ & 919 & $158(17,2)$ & $1,01(0,78-1,30)$ & & 780 & $131(16,8)$ & & \\
\hline$\geq 35$ & 403 & $72(17,9)$ & 1,0 & & 365 & $53(14,5)$ & & \\
\hline Escolaridade materna (anos) & & & & & & & & \\
\hline$<4$ & 861 & $205(23,8)$ & $1,65(1,21-2,27)$ & $0,006^{\mathrm{e}}$ & - & - & & - \\
\hline $4-7$ & 644 & $138(21,4)$ & $1,55(1,15-2,09)$ & & - & - & & - \\
\hline$\geq 8$ & 547 & $63(11,5)$ & 1,0 & & - & - & & - \\
\hline & & & Nível proxima & & & & & \\
\hline Grupo E & & & & & & & & \\
\hline Idade da criança (meses) & & & & & & & & \\
\hline $0-11$ & 454 & $113(24,9)$ & $1,57(1,27-1,94)$ & & 296 & $49(16,6)$ & & \\
\hline $12-23$ & 410 & $112(27,3)$ & $1,73(1,41-2,12)$ & $<0,001^{f}$ & 347 & $97(28,0)$ & & $<0,001^{\dagger}$ \\
\hline $24-59$ & 1.188 & $181(15,2)$ & 1,0 & & 953 & $142(14,9)$ & & \\
\hline
\end{tabular}

a Razões de prevalências ajustadas.

${ }^{\mathrm{b}}$ Ajustados para a variável do nível A.

${ }^{c}$ Ajustado para as variáveis dos níveis A e B.

${ }^{\mathrm{d}}$ Ajustados para as variáveis dos níveis A, B e C.

e Ajustado para as variáveis dos níveis A, B, C e D.

${ }^{\dagger}$ Ajustado para as variáveis dos níveis A, B, C, D e E. 


\section{DISCUSSÃO}

Os problemas de saúde, quando considerados em nível populacional e como processos individuais ou clínicos, envolvem múltiplos fatores em sua determinação. Assim, as variações que ocorrem no espaço geográfico entre grupos biológicos e sociais e, sobretudo, em escala temporal, como as tendências históricas do processo saúde ou doença, representam desfechos de caráter multifatorial, combinando um jogo mais ou menos complexo de causas que atuam em interação. Portanto, não existe um modelo único de fatores explicativos que possa ser universalmente aplicado em territórios diferentes, em tempos distintos e em agrupamentos humanos que evoluem com características próprias, ainda que influenciadas por processos globais?

Esses fundamentos conceituais estão bem ilustrados quando analisa-se, como no caso deste estudo, o comportamento epidemiológico das diarreias em menores de cinco anos no estado de Pernambuco, em um espaço de tempo relativamente breve e recente, ou seja, nos anos de $1997 \mathrm{e}$ 2006. Em uma abordagem descritiva, ficaram evidenciadas mudanças geográficas marcantes na prevalência do problema, sua distribuição espacial e socioambiental, suas demandas de hospitalização e sua participação (ou impacto) na composição das causas de mortes. Trata-se de uma cadeia de eventos que, além de sua significação própria, como representação de uma nosologia específica, insere-se no contexto de um processo mais geral: a história recente da transição ${ }^{18}$.

Fica claro tanto em termos de análises univariadas, quanto em termos de compreensão das diarreias sob uma abordagem multivariada, as importantes diferenças que demarcam o trânsito epidemiológico do problema em um grupo específico de hospedeiros: crianças menores de cinco anos.

Nessa perspectiva, destacam-se as variações consideráveis registradas no elenco dos cinco grupos de fatores e suas respectivas subcategorias, compostas de três níveis de análises multivariadas. O ingresso ou a exclusão de muitas variáveis por critérios mais estatísticos que conceituais podem, ao lado de outras limitações que serão oportunamente ressaltadas, representar uma possível impropriedade de abordagem metodológica. Entretanto, a impressão que prevalece é que a diarreia, como uma nosografia traçadora de situações de saúde, expressa de forma sensível o processo dinâmico de um conjunto de fatores que se articulam desde o nível estrutural da sociedade até a instância familiar ou individual de sua determinação, o que provoca as mudanças de resultados analíticos no período avaliado.

Partindo dos resultados das análises uni e multivariadas tratadas em nosso estudo, ressaltando dois aspectos (a notável redução à prevalência de diarreia na Região Metropolitana do Recife e a relação que o abastecimento ou tratamento de água significou, estatisticamente, na diminuição do risco deste problema em crianças), seria possível presumir dois eventos ecológicos concordantes. O primeiro refere-se aos grandes avanços da cobertura do abastecimento público de água na Região Metropolitana do Recife nos últimos 10 anos, ao contrário do ocorrido no restante do estado, seja em espaços urbanos ou rurais. O segundo refere-se à crise de suprimento de água tratada que se agravou de forma significativa, de tal maneira que a solução há vários anos consiste na construção de dois grandes sistemas de adutoras no Sertão e no Agreste, ainda em andamento, para as populações urbanas e rurais através da transposição do Rio São Francisco ${ }^{19,20}$. São observações compatíveis com a hipótese explicativa aqui sugerida e seriam duas cartografias separadas pelas condições básicas de saneamento (água, esgoto e lixo).

No Brasil, estima-se que 36 milhões de pessoas ainda não tenham acesso à água potável. Além disso, menos da metade da população brasileira não conta com coleta de lixo e apenas $38 \%$ dos esgotos não são tratados, o que colaborou com as 212.000 internações em 2011 por diarreia em menores de cinco anos no país ${ }^{21}$. Estudo realizado na Bahia para avaliar o impacto do Programa Água para Todos, que contemplou 224 municípios, concluiu que os que tiveram cobertura de $10 \%$ apresentaram uma redução de $14 \%$ na mortalidade por diarreia em menores de cinco anos e em $6 \%$ nas internações hospitalares, quando comparados aos municípios sem cobertura ou aos que apresentaram cobertura menor ${ }^{22}$. 
Ao analisar os modelos finais hierarquizados dos anos em estudo, 1997 e 2006, destacamos algumas variáveis que se mantiveram associadas à diarreia nos dois períodos, como a idade materna, a idade da criança e o número de pessoas por cômodo. Em outras situações, ressaltam-se variáveis que não constavam no modelo ajustado, como abastecimento e tratamento da água em 1997, mas que ingressaram em 2006. Por outro lado, alguns grupos de variáveis que figuram no modelo final de 1997, como o destino do lixo e a disponibilidade de geladeira no domicílio, que integrava o modelo de risco em 1997, deixaram de participar em 2006.

A idade da mãe tem se mostrado frequentemente associada à prevalência de diarreia ${ }^{23,24}$. A associação da doença nos filhos de mães mais jovens pode ser atribuída à maior probabilidade de conceberem filhos com baixo peso, além da inexperiência materna nos cuidados com as crianças e maiores dificuldades em alimentar adequadamente seus filhos, inclusive com tendência ao desmame precoce ${ }^{25,26}$.

A idade da criança implicou em um risco mais elevado nos grupos de zero a 11 meses e 12 a 24 meses, em comparação com as crianças de maior idade. Esta é uma tendência universal, com várias razões biológicas, culturais e socioambientais para esse risco aumentado ${ }^{27}$.

Apesar do aumento da renda no período dos nove anos (1997-2006), por conta do aumento do salário mínimo, programa de transferência de renda e ao próprio desenvolvimento econômico do país, em Pernambuco, ainda se observa um contingente considerável de casos em que a renda familiar per capita está abaixo de 0,5 (meio) salário mínimo, condição que alcançou um papel significativo no modelo multivariado. A baixa renda favorece a ocorrência de fatores predisponentes ou agravantes das doenças diarreicas em função do baixo nível de educação e condições precárias de vida, constituindo o chamado ecossistema de pobreza ${ }^{28,29}$. Como exemplo, a variável "número de pessoas por cômodo" sugere que a alta aglomeração de pessoas pode favorecer a precariedade de condições de higiene e a contaminação dos alimentos e da água. Da mesma forma, essas condições adversas se aplicam ao tratamento da água de beber, que passou a assumir papel significativo na análise mais recente em 2006.

Outras características das habitações, famílias e crianças não ressaltadas nos modelos simples ou ajustados deixam de ser aqui debatidas, porém não significa que não possam ter um papel de proteção ou risco em outras circunstâncias ou contexto.

O estudo apresenta limitações que podem afetar em alguns aspectos sua validade interna ou externa. Os dados não foram gerados a partir de um estudo previamente desenhado para analisar os fatores de risco de diarreia. Além disso, persistem dúvidas sobre resultados estatísticos inesperados como no caso do tratamento da água, sem uma explicação conveniente, e há também limitações epidemiológicas próprias dos inquéritos de prevalência com registros simultâneos de causa e efeito. Adicionalmente, o ajuste de resultados em função do peso amostral não foi realizado, uma vez que o estudo inicial da série histórica (1991) não contemplou esses aspectos no estado de Pernambuco ${ }^{9}$ ou em quaisquer dos oito estados do Nordeste pesquisados; são, portanto, restrições que não podem ser corrigidas.

Pela abrangência de fatores avaliados, originalidade da abordagem, dimensão temporal de suas tendências e configuração epidemiológica de suas observações e análises, este estudo pode contribuir para a proposição de alternativas políticas e programáticas para o controle do problema, bem como motivar a realização de novos inquéritos.

Em nível de políticas públicas, apesar de mudanças em termos de pessoas, sequências temporais e espaços geográficos, as diarreias continuam em uma escala importante no elenco de prioridades do poder governamental. Ao lado dessas variações epidemiológicas básicas, foram identificados os fatores isolados ou combinados na sua determinação, o que sinaliza novas indicações estratégicas para seu efetivo controle. 


\section{REFERÊNCIAS}

1. The United Nations Children's Fund; World Health Organization. Diarrhoea: why children are still dying and what can be done. New York: UNICEF/WHO; 2009 [citado 21 ago 2013]. Disponível em: www.unicef.org.?media/files/Final_Diarrhoea-Report-October-2009-final.pdf

2. Mendes PSA, Ribeiro Jr HC, Mendes CMC. Temporal trends of overall mortality and hospital morbidity due to diarrheal disease in Brazilian children younger than 5 years from 2000 to 2010. J Pediatr (Rio J). 2013;89(3):315-25. https://doi.org/10.1016/j.jped.2012.10.002

3. Walker CLF, Perin J, Aryee MJ, Boschi-Pinto C, Black RE. Diarrhea incidence in low-and-middle income countries in 1990 and 2010: a systematic review. BMC Public Health. 2012;12:220. https://doi.org/10.1186/1471-2458-12-220

4. Wardlaw T, Salama P, Brocklehurst C, Chopra M, Manso E. Diarrhoea: why children are still dying and what can be done. Lancet. 2010;375(9718):870-2 . https://doi.org/10.1016/S0140-6736(09)61798-0

5. Black RE, Cousens S, Johnson HL, Lawn JE, Rudan I, Bassan DG, et al. Global, regional and national causes of child mortality in 2008: a systematic analysis. Lancet. 2010;375(9730):1969 87. https://doi.org/10.1016/S0140-6736(10)60549-1

6. Rouquayrol MZ, Goldbaum M, Santana EWP. Epidemiologia, história natural e prevenção de doenças. In: Rouquayrol MZ, Gurgel M. Epidemiologia e saúde. 7.ed. Rio de Janeiro: MedBook; 2013. p.11-24.

7. UNICEF. Situação mundial da infância 2012: crianças em um mundo urbano. New York; 2012 [citado 21 ago 2013]. Disponível em: https://www.unicef.org/brazil/pt/PT-BR_SOWC_2012.pdf

8. Kessner DM, Kalk CE, Singer J. Assessing health quality: the case for tracers. N Engl J Med. 1973;288(4):189-94. https://doi.org/10.1056/NEJM197301252880406

9. Secretaria da Saúde do Estado de Pernambuco; UNICEF. I Pesquisa Estadual de Saúde e Nutrição: crianças e adolescentes em Pernambuco: saúde, educação e trabalho. Recife; 1991.

10. Secretaria da Saúde do Estado de Pernambuco. II Pesquisa Estadual de Saúde e Nutrição: saúde, nutrição, alimentação e condições socioeconômicas no Estado de Pernambuco. Recife; 1998

11. Schramm JMA, Oliveira AF, Leite IC, Valente JG, Gadelha AMJ, Portela MC, et al. Transição epidemiológica e o estudo da carga de doença no Brasil. Cienc Saude Coletiva. 2004;9(4):897908. https://doi.org/10.1590/S1413-81232004000400011

12. Araújo JD. Polarização epidemiológica no Brasil. Epidemiol Serv Saude. 2012;21(4)533-8. https://doi.org/10.5123/S1679-49742012000400002

13. Secretaria da Saúde do Estado de Pernambuco. III Pesquisa Estadual de Saúde e Nutrição: situação alimentar, nutricional e de saúde no Estado de Pernambuco: contexto socioeconômico e de serviços: relatório final. Recife; 2007.

14. Ministério da Saúde(BR), Secretaria de Assistência à Saúde, Coordenação de Saúde MaternoInfantil. Assistência e controle das doenças diarreicas. Brasília (DF); 1993 [citado 21 ago 2013]. Disponível em: http://bvsms.saude.gov.br/bvs/publicacoes/partes/doencas_diarreicas1.pdf.

15. WHO Multicentre Growth Reference Study Group. WHO child growth standards: length/ height-for-age, weight-for-age, weight-for-length, weight-for-height and body mass index-for-age: methods and development. Geneva: WHO; 2006.

16. World Health Organization. WHO Anthro: software for assessing growth and development of the world's children. Version 3.2.2. Geneva: WHO; 2011 [citado 6 jun 2011] Disponível em: http://www.who.int/childgrowth/software/en/

17. Fuchs SC, Victora CG, Fachel J. Modelo hierarquizado: uma proposta de modelagem aplicada à investigação de fatores de risco para diarréia grave. Rev Saude Publica. 1996;30(2):168-78. https://doi.org/10.1590/S0034-89101996000200009

18. Batista Filho M, Souza Al, Miglioli TC, Santos MC. Anemia e obesidade: um paradoxo de transição nutricional brasileira. Cad Saude Publica. 2008;24 Supl 2:s247-57. https://doi.org/10.1590/S0102-311X008001400010

19. Instituto Trata Brasil. Ranking do saneamento: resultados com base no SNIS 2012. São Paulo; 2014 [citado 11 fev 2015]. Disponível em: http://www.tratabrasil.org.br/datafiles/estudos/ ranking/relatorio-completo-2014.pdf

20. Instituto de Pesquisa Econômica Aplicada (IPEA). Transposição do Rio São Francisco: análise de oportunidade do projeto. César Nunes Castro. Rio de Janeiro; 2011 [citado 11 abr 2012]. Disponível em: http://repositorio.ipea.gov.br/bitstream/11058/1418/1/TD_1577.pdf 
21. Instituto Trata Brasil. Saneamento básico: há dinheiro e tecnologia, mas faltam projetos e vontade política. São Paulo; c2013 [citado 20 dez 2014]. Disponível em: http://www.tratabrasil. org.br/saneamento-basico-ha-dinheiro-e-tecnologia-mas-faltam-projetos-e-vontade-politica

22. Rassela D. Impacto do Programa Água para Todos (PAT) sobre a morbi-mortalidade por diarreia em crianças do Estado da Bahia, Brasil. Cad Saude Publica. 2013;29(1):40-50. https://doi.org/10.1590/S0102-311X2013000100006

23. Sinmegn MT, Asres AG, Shimeka TA. Determinants of childhood diarrhea among underfive children in Benishangul Gumuz Regional State, North West Ethiopia. BMC Pediatr. 2014;14:102. https://doi.org/10.1186/1471-2431-14-102

24. Finlay JE, Ozaltin E, Canning D. The association of maternal age with infant mortality, child antropometric failure, diarrhoea and anemia for first birhs: evidence from 55 low-and middle-income countries. BMJ Open. 2011;1(2):e000226. https://doi.org/10.1136/bmjopen-2011-000226

25. Picot J, Hartwell D, Harris P, Mendes D, Clegg AJ, Takeda A. The effectiveness of interventions to treat severe acute malnutrition in young children: a systematic review. Health Technol Assess. 2012;16(19):1-316. https://doi.org/10.3310/hta16190

26. Guimarães AMDN, Bettiol H, Souza L, Gurgel RQ, Almeida MLD, Ribeiro ERO, et al. Gravidez na adolescência é fator de risco para o baixo peso ao nascer? Rev Saude Publica. 2013;47(1):119. https://doi.org/10.1590/S0034-89102013000100003

27. Mehal JM, Esposito DH, Holman RC, Tate JE, Callinan LS, Parashar UD. Risk factors for diarrheaassociated infant mortality in the United States, 2005-2007. Pediatr Infect Dis J. 2012;31(7):71721. https://doi.org/10.1097/INF.0b013e318253a78b

28. Das SK, Faruque AS, Chisti MJ, Malek MA, Salam MA, Sack DA. Changing trend of persistent diarrhoea in young children over two decades: observations from a large diarrhoeal disease hospital in Bangladesh. Acta Paediatr. 2012;101(10):452-7. https://doi.org/10.1111/j.1651-2227.2012.02761.x

29. Barreto ML, Teixeira MG, Bastos Fl, Ximenes RAA, Barata RB, Rodrigues LC. Sucessos e fracassos no controle de doenças infecciosas no Brasil: o contexto social e ambiental, políticas, intervenções e necessidades de pesquisa. London: The Lancet; 2011 [citado 15 jul 2014]. p.47-60. (Saúde no Brasil, 3). Disponível em: http://www.ins.gob.pe/repositorioaps/0/0/jer/ maestria_2012/Art\%C3\%ADculo\%202.pdf

Contribuição dos Autores: Concepção e planejamento do estudo: MJOBV, MBF. Coleta dos dados, análise e interpretação dos dados: MJOBV, MBF, PICL, JNF. Elaboração e revisão do manuscrito: MJOBV, MBF, AR. Todos os autores aprovaram a versão final do artigo e assumem a responsabilidade pública pelo conteúdo.

Conflito de Interesses: Os autores declaram não haver conflito de interesses. 